\title{
Estructura tributaria será más regresiva si se aprueba la reforma ${ }^{1}$
}

\author{
Red Colombiana por la Justicia Tributaria
}

Recibido: 20 de noviembre de 2016

Aprobado: 19 de diciembre de 2016

\section{Red Colombiana por la Justicia Tributaria. (2016). Estructura tributaria será más regresiva si se aprueba la reforma. Activos, 27, 19-32.}

Clasificación JEL: H20, H23, H25.

\section{Resumen}

El presente documento realiza un análisis a las principales propuestas realizadas por el Gobierno colombiano en el marco de la propuesta de reforma tributaria, la cual fue presentada al público en el mes de octubre de 2016 y finalmente aprobada en diciembre del mismo año como Ley 1819. Dicho análisis plantea la inconveniencia de las principales líneas de propuesta de la reforma, en un contexto económico particularmente complejo, y donde las

1 Documento elaborado por la Red Colombiana por la Justicia Tributaría, con el apoyo de Christian Aid, con objeto del debate de la reforma tributaria 2016, aprobada como Ley 1819 de 2016. Este documento se publica por el mérito de su estructura de análisis y porque constituye la memoria sobre las consecuencias que generará dicha reforma sobre el consumo y el bienestar de los ciudadanos.

2 La Red Colombiana por la Justicia Tributaria, es una organización de la sociedad civil, constituida por un conjunto de académicos y expertos en temas tributarios y fiscales, que hacen un permanente seguimiento a las decisiones económicas del Gobierno colombiano en materia fiscal, para mayor información sobre sus actividades y otros documentos ver www.justiciatributaria.co 
nuevas condiciones de impuestos como el Impuesto al Valor Agregado - IVA, reducirá notoriamente la capacidad de consumo de las familias, mientras que en impuestos directos como renta y patrimonio especialmente los pagados por los grandes capitales se obtienen alivios sustanciales.

El documento concluye argumentando que la propuesta del Gobierno aumentará la regresividad del sistema tributario colombiano y empeorará las condiciones de vida, especialmente la de los hogares de menores ingresos.

\title{
Palabras clave
}

Política tributaria, reforma tributaria, regresividad, Impuesto al Valor Agregado.

Red Colombiana por la Justicia Tributaria. (2016). Tax structure
will be more regresiveness if approved the reform. Activos, 27, 19-32.

\begin{abstract}
This paper make an analysis of principal proposals realized by Colombian Govermentin the frame or Tax Reform Initiative, presented to the public in October of 2016, and finally approved in december of same year, under the 1819 law. These analysis raises the inconvenience of main lines proposed by Tax Reform, in a economical context particularly complex, and where new conditions in Taxes like Added-Value - AVT, will reduce notoriously the capacity of family's consumption, while in direct taxes, like Income and Heritage, especially payed by great capitals obtain substancial reliefs.

The paper concludes that the goverment proposed increasing the regresivity of colombian tax system, worse the life conditions, especially of low income households.
\end{abstract}




\section{Keywords}

Tax policy, tax reform, regresivity, Added-Value Tax.

Red Colombiana por la Justicia Tributaria. (2016). La structure fiscale sera plus régressive si la réforme est approuvée. Activos, 27, 19-32.

\section{Résumé}

Ce document analyse les principales propositions faites par le gouvernement colombien dans le cadre de la proposition de réforme fiscale qui a été présentée au public en octobre 2016, et qui a finalement été approuvée en décembre de la même année comme la Loi 1819. Cette analyse souligne l'inopportunité des grandes lignes de cette proposition de réforme dans un contexte économique particulièrement difficile, et où les nouvelles conditions de certains impôts telles que la taxe sur la valeur ajoutée - TVA, réduira sensiblement le pouvoir d'achat des ménages, alors que les impôts directs comme l'impôt sur le revenu et sur la richesse, et ceux qui sont particulièrement payés par les grands capitaux ont été substantiellement baissés.

Le document conclut en argumentant que la proposition du gouvernement augmentera la régressivité du système fiscal colombien et aggravera les conditions de vie, et en particulier celles des ménages à faible revenu.

\section{Mots-clés:}

Politique fiscale, réforme fiscale, régressivité, Taxe sur la Valeur Ajoutée-TVA. 


\section{Regresividad, regresividad, regresividad... El dogma del Gobierno}

La propuesta de reforma tributaria que el presidente Juan Manuel Santos y el ministro Mauricio Cárdenas presentaron al Congreso de la República es el resultado del desespero por resolver, en forma inmediata, el déficit fiscal y de la aceptación de la presión de las calificadoras de riesgo y de la OCDE para mantener el grado de inversión, a costa de ahogar la economía por la vía de reducir el nivel de vida de los hogares de ingresos bajos y medios.

Los platos rotos del manejo irresponsable de la economía durante los últimos años, en los cuales se dilapidó la bonanza petrolera, se avanzó en la destrucción del aparato productivo y el empleo de calidad y se colmó de beneficios a las transnacionales, los pagarán la ciudadanía más necesitada. El resultado previsible es la profundización de la desigualdad por cuenta de una estructura tributaria regresiva que aumenta los impuestos indirectos y reduce los directos. La propuesta de reforma hace caso omiso de que los ingresos estatales han disminuido por cuenta de la destrucción del aparato productivo, como resultado de decisiones como la implementación de múltiples TLC. En la exposición de motivos no se señala la gravedad de que el déficit está ligado a un aumento del endeudamiento externo que llega a un 41,5\% del PIB y de la deuda pública que llega a un 54,1 \% del PIB.

El mismo Gobierno reconoce descaradamente que los tributos directos "deben extinguirse" y las finanzas públicas deben "generar nuevos ingresos, en particular los provenientes del IVA" (Ministerio de Hacienda, 2015, p. 7). En efecto, las cifras muestran que para el año 2017 el aumento del IVA y los demás impuestos indirectos recaudarían $\$ 9,1$ billones, $1 \%$ del PIB, (incluye IVA, impuesto al consumo y combustibles), mientras el impuesto a la renta caería en $\$ 1,9$ billones ( $0,2 \%$ del PIB). Similar comportamiento se presentará en el 2018, cuando se espera que en impuestos indirectos se recaude $\$ 10,8$ billones y en renta se dejen de percibir recursos por $\$ 1,2$ billones, como se puede ver en la tabla 1. 
De esta forma, los impuestos indirectos explicarían la totalidad del nuevo recaudo, por lo que la principal discusión sobre la próxima reforma se centra en este punto. Los demás asuntos serán negociados en el Congreso de la República.

Solo a un Gobierno de la naturaleza del de Santos podría ocurrírsele que para resolver los graves problemas de desigualdad del país, que lo ubican como uno de los diez con mayor concentración de la riqueza en el planeta, se debe acudir a aumentar los impuestos indirectos como el IVA y disminuirlos a los grandes capitales que operan en Colombia.

Tabla 1. Recaudo adicional del Gobierno Nacional Central por tipo de impuesto esperado con reforma 2017-2022.

(\$ miles de milllones)

\begin{tabular}{l|c|c|c|c|c|c}
\hline Concepto & 2007 & 2018 & 2019 & 2020 & 2021 & 2022 \\
\hline IVA e impuesto al consumo & 8,163 & 9,815 & 10,755 & 11,843 & 12,833 & 13,868 \\
\hline GMF & 0 & 0 & 2,244 & 4,836 & 7,830 & 11,358 \\
\hline Renta & $-1,890$ & $-1,174$ & 1,722 & 326 & 354 & 382 \\
\hline Combustibles & 918 & 1,034 & 1,191 & 1,371 & 1,574 & 1,789 \\
\hline Subtotal & 7,191 & 9,676 & 15,912 & 18,376 & 22,591 & 27,397 \\
\hline Gestión DIAN & 0 & 0 & 3,633 & 5,035 & 6,646 & 7, \\
\hline Total & 7,191 & 9,676 & 19,544 & 23,411 & 29,237 & 34,570 \\
\hline
\end{tabular}

\section{Más impuestos indirectos que pagarán los menos favorecidos}

De acuerdo con la propuesta de reforma, el IVA pasaría de $16 \%$ al 19 $\%$, incluyendo en este aumento al $60 \%$ de bienes de la canasta familiar que ya están gravados. Además, mantiene el IVA del $5 \%$ a otros bienes que este Gobierno gravó en la reforma de 2012, como trigo, café, chocolate, aceites vegetales, pastas alimenticias y hasta el salchichón; ahora se suman los diarios y publicaciones periódicas e impresos en esta categoría.

En un ejercicio hecho por Justicia Tributaria, tomando una canasta de 15 productos básicos que hoy en día están gravados al $16 \%$ y se verían 
afectados con el aumento de la tarifa general del IVA al $19 \%$, se puede demostrar que tan solo en estos bienes habría un incremento de \$3.533 pesos, como se observa en el cuadro 2 , siendo productos como el jabón de baño, el papel higiénico, las salchichas, las hojuelas, de los productos más afectados y que son, la mayoría de ellos, de uso básico.

Tabla 2. Análisis de precios de 15 productos gravados al $16 \%$ y su impacto de subir el IVA al $19 \%$.

\begin{tabular}{|c|c|c|c|c|c|c|}
\hline $\begin{array}{l}\text { Producto / cifras } \\
\text { en pesos }\end{array}$ & $\begin{array}{l}\text { Precio } \\
\text { actual }\end{array}$ & $\begin{array}{c}\text { IVA } \\
(16 \%)\end{array}$ & $\begin{array}{l}\text { Precio sin } \\
\text { IVA }\end{array}$ & $\begin{array}{c}\text { IVA } \\
(19 \%)\end{array}$ & $\begin{array}{c}\text { Precio } \\
\text { proyectado }\end{array}$ & Diferencia \\
\hline Jabón para cuerpo x6 & 14.100 & 2.256 & 11.844 & 2.679 & 14.523 & 423 \\
\hline Crema dental x2 & 8.400 & 1.344 & 7.056 & 1.596 & 8.652 & 252 \\
\hline Papel higiénico x12 & 13.900 & 2.224 & 11.676 & 2.641 & 14.317 & 417 \\
\hline Detergente x $3 \mathrm{~kg}$ & 12.600 & 2.016 & 10.584 & 2.394 & 12.978 & 378 \\
\hline Salchichas & 6.200 & 992 & 5.208 & 1.178 & 6.386 & 186 \\
\hline $\begin{array}{l}\text { Aceite de cocina } \\
\mathrm{x} 1000 \mathrm{~cm}^{3}\end{array}$ & 4.150 & 664 & 3.486 & 789 & 4.275 & 125 \\
\hline Spaghetti x $2 \mathrm{~kg}$ & 10.000 & 1.600 & 8.400 & 1.900 & 10.300 & 300 \\
\hline Atún x2 latas & 7.800 & 1.248 & 6.552 & 1.482 & 8.034 & 234 \\
\hline $\begin{array}{l}\text { Limpiapisos x1000 } \\
\mathrm{ml}\end{array}$ & 2.300 & 368 & 1.932 & 437 & 2.369 & 69 \\
\hline $\begin{array}{l}\text { Hojuelas azucaradas } \\
\text { x } 900 \mathrm{~g}\end{array}$ & 10.350 & 1.656 & 8.694 & 1.967 & 10.661 & 311 \\
\hline $\begin{array}{l}\text { Chocolate en polvo } \\
\mathrm{x} 400 \mathrm{~g}\end{array}$ & 6.400 & 1.024 & 5.376 & 1.216 & 6.592 & 192 \\
\hline $\begin{array}{l}\text { Barra de jabón para } \\
\text { ropa x } 3\end{array}$ & 3.000 & 480 & 2.520 & 570 & 3.090 & 90 \\
\hline Suavizante x $2000 \mathrm{ml}$ & 5.200 & 832 & 4.368 & 988 & 5.356 & 156 \\
\hline $\begin{array}{l}\text { Salsa de tomate } \\
\mathrm{x} 600 \mathrm{~g}\end{array}$ & 9.660 & 1.546 & 8.114 & 1.835 & 9.950 & 290 \\
\hline Lavaloza x1000 ml & 3.700 & 592 & 3.108 & 703 & 3.811 & 111 \\
\hline TOTAL & 117.760 & 18.842 & 98.918 & 22.374 & 121.293 & 3.533 \\
\hline
\end{tabular}

Fuente: elaboración propia con base en datos de Supermercados D1 y Colsubsidio para el mes de octubre de 2016. 
Adicionalmente, teniendo en cuenta los ingresos de una persona que devenga mensualmente $\$ 1^{\prime} 500.000$-quien comenzará a pagar retefuente ${ }^{3}$ si se aprueba la reforma- los más afectados con el aumento del IVA serán los hogares de ingresos bajos y medios, porque tendrían que destinar una mayor porción de su ingreso a la adquisición de estos bienes, en comparación con hogares de ingresos altos.

En la tabla 3 se presentan los resultados en los cuales el trabajador con ingresos de $\$ 1$ '500.000 mensuales, con el aumento del IVA al $19 \%$, tendría que destinar $0,24 \%$ más de su ingreso para adquirir los mismos productos, mientras una persona que gane más de $\$ 16^{\prime} 000.000$ mensuales tendría que destinar solo $0,02 \%$ más, una diferencia de 12 veces. En total, un trabajador debe destinar el $8 \%$ de su ingreso mensual para adquirir estos 15 productos con IVA del $19 \%$, mientras una persona de ingresos altos tan solo destina el $0,72 \%$ para el mismo propósito. De esta forma el aumento del IVA aumentará la inequidad.

Tabla 3. Impacto de aumento del IVA por nivel de salarios.

\begin{tabular}{l|c|c|c|c}
\hline Cifras en pesos & $\begin{array}{c}\text { Salario } \\
\text { mensual }\end{array}$ & $\begin{array}{c}\text { Destinación } \\
\text { mercado } \\
\text { (antes de } \\
\text { reforma) }\end{array}$ & $\begin{array}{c}\text { Destinación } \\
\text { mercado } \\
\text { después de } \\
\text { reforma) }\end{array}$ & Diferencia \\
\hline $\begin{array}{l}\text { Ministros } \\
\text { (promedio) }\end{array}$ & 16.803 .090 & $0,70 \%$ & $0,72 \%$ & $0,02 \%$ \\
\hline $\begin{array}{l}\text { Asalariado clase } \\
\text { media }\end{array}$ & 1.500 .000 & $7,85 \%$ & $8,09 \%$ & $0,24 \%$ \\
\hline
\end{tabular}

Fuente: elaboración propia con base en datos de la escala salarial presentada por ElEspectador (30 de junio de 2016).

También se encuentra en la reforma la limitación de la exclusión del IVA para la compra de celulares y computadores y la imposición de un impuesto al consumo de datos móviles del $4 \%$, los cuales van en contravía del discurso

3 Artículo 16 del Proyecto de Reforma. Pagarán retefuente ingresos desde \$1’487.650, actualmente pagan desde \$2'826.535 (artículo 383 del Estatuto Tributario). 
gubernamental de promover la inserción del país a la economía del conocimiento, las tecnologías modernas y la cuarta revolución industrial. En esta categoría, el Gobierno de Santos propone también gravar a las editoriales con un impuesto de renta de $9 \%$ y $32 \%$, que afectaría fundamentalmente a quienes están intentando crear conocimiento en el país y se beneficiaría la importación de textos, un duro golpe a la producción editorial, intelectual y cultural del país.

Además, los combustibles también se verán afectados con la reforma, ya que la propuesta contempla un impuesto adicional a la gasolina de \$135 adicionales por galón (tabla 4), denominado 'impuesto al carbono', que se sumarían a los más de $\$ 1.000$ que se pagan hoy en día por galón de gasolina corriente con el impuesto nacional a la gasolina y ACPM, que recae sobre los hogares de ingresos bajos y medios que son los mayores consumidores de combustibles, porque en estos estratos se ubica cerca del $92 \%$ del parque automotor. En este mismo sentido, preocupa la propuesta de crear una contribución parafiscal al combustible para financiar el Fondo de Estabilización de Precios de los Combustibles (FEPC), que ya ha sido declarado inconstitucional (Sentencia C-726/15, Corte Constitucional) y que tiene un déficit de $\$ 5$ billones a 2015.

Tabla 4. Impuesto al carbono por galón y tipo de combustible.

\begin{tabular}{l|c|c}
\hline Combustible fósil & Unidad & Tarifa/unidad \\
\hline Gas licuado de petróleo & Galón & $\$ 95$ \\
\hline Gasolina & Galón & $\$ 135$ \\
\hline Kerosene y Jet Fuel & Galón & $\$ 148$ \\
\hline ACPM & Galón & $\$ 152$ \\
\hline Fuel oil & Galón & $\$ 177$ \\
\hline
\end{tabular}

Fuente: proyecto de ley reforma tributaria, artículo 208. 


\section{Un Gobierno al servicio de las transnacionales}

Las tres mil más poderosas empresas pagarían menos impuesto a la renta $y$, las empresas en general, pagarían menos impuesto a la renta, bajando en promedio del $40 \%$ al $32 \%$. Adicionalmente, gran parte de las exenciones tributarias y demás beneficios que gozan las más grandes se mantienen e incluso se crean nuevos, como el de "costo fiscal de las inversiones" para los gastos de evaluación y exploración del sector minero-energético.

Sin embargo, se mantiene la regresividad del impuesto a la renta, porque a diferencia de los países con que Colombia compite en los negocios globales, no hay tarifas diferenciadas entre pequeñas, medianas y grandes empresas. Además, no se tiene en cuenta el nivel de desigualdad que en las empresas es más escandaloso que en las personas. En un estudio de ACOPI Bogotá (Suárez y Martínez, 2016), se observa que el nivel de concentración -teniendo en cuenta los excedentes brutos de explotación- llega a un Gini de 0,822; por tamaño y valor agregado para los sectores industriales en Colombia llega al 0,7542; por tamaño y activos para los sectores industriales llega al 0,8334. Estos resultados deberían ser la base para determinar una tarifa diferencial. Una tarifa uniforme y homogénea, como se propone con el Impuesto Único Empresarial (IUE), no tiene en cuenta la alta concentración que existe en las empresas de Colombia y grava por igual a las pequeñas y a las grandes.

Aunque el Gobierno ha sido insistente en afirmar que los dividendos pagarán impuestos, una propuesta progresiva, la realidad es que la reforma tributaria establece que solo se hará si el pago de dividendos recae en cabeza de una persona natural, lo que en la práctica permite que se paguen dividendos sin impuestos a los socios de compañías que utilizan empresas para recibir estos ingresos.

En Colombia viene haciendo carrera la idea de que las empresas deberían pagar menos impuestos y que estos deben recaer sobre personas naturales. Sin embargo, se ignora que la administración tributaria tiene una capacidad limitada para establecer quién es el beneficiario real de las actividades de empresas extranjeras en el país, los directivos de compañías 
extranjeras -en general- reciben sus sueldos en las casas matrices y sus gastos de sostenimiento en Colombia son cargados como gastos de las empresas, prácticas que imposibilitan cobrar impuestos a las personas naturales que derivan sus ingresos del trabajo con este tipo de empresas y erosionan la base tributaria.

Además, las transnacionales utilizan los bienes públicos en materia de seguridad e institucionalidad, por lo que deben pagar lo justo en retribución al Estado.

Pero como es esta la lógica que se está imponiendo en la tributación nacional, los trabajadores colombianos deben asumir una mayor carga. Por eso la reforma propone gravar a las persona naturales desde \$2’479.000 de ingresos mensuales con una tarifa del $10 \%$ y pagar retefuente desde $\$ 1$ '487.650, un ingreso insuficiente para que una familia mejore su calidad de vida, pero que la dupla Santos-Cárdenas considera de millonarios. Esto ocurre porque el actual Gobierno, como también lo ha reconocido, presentó una reforma tributaria dictada por la Organización para la Cooperación y el Desarrollo Económico (OCDE), que sigue lineamientos de los países ricos de ese club, a los que Colombia no se parece en materia social ni económica.

En el caso del monotributo, a pesar de ser opcional, este es el primer paso para gravar a los pequeños comerciantes. Además, en una clara violación al principio de equidad, tenderos y salones de belleza -entre otros- tendrían que pagar entre $\$ 40.000$ y $\$ 80.000$ mensuales en impuestos que se cobran sobre sus ventas, mientras al resto de empresas más grandes se les cobra sobre las utilidades.

\section{La reforma atenta contra el principio constitucional de libertad de asociación}

Por el lado de las Entidades sin Ánimo de Lucro (ESAL), el Gobierno propone un control a la actividad teniendo exclusivamente criterios tributarios y no sobre la verdadera naturaleza de las organizaciones. Impone, 
por ejemplo, la obligación de que la cooperación internacional tenga que registrase ante la Agencia Presidencial para la Cooperación (APC Colombia), y no diferencia entre las pequeñas que cumplen una misión social y las grandes, asestando un duro golpe a la Constitución al violar la libertad de asociación, en una clara persecución a quienes realizan vigilancia, control y veeduría a las políticas gubernamentales. Además, todas serán consideradas contribuyentes del régimen ordinario y las no contribuyentes serán obligadas a declarar ingresos y patrimonio, aumentando el control estatal sobre las mismas a excepción solamente de las Juntas de Acción Comunal.

Con esta reforma, el Estado da un paso más en la lógica de libre mercado en la cual todas las actividades de una sociedad deben ser consideradas con ánimo de lucro, como si fueran bienes o servicios de mercado, incluyendo aspectos tan sensibles para el desarrollo social como los sindicatos, las cooperativas de pequeños productores y comerciantes, las Juntas de Acción Comunal y hasta los bomberos.

\section{Los otros temas de la reforma que afectan a la población}

Por el lado del fortalecimiento administrativo, a pesar de que se le dedican 14 artículos de la reforma, no aporta nada nuevo cuando se refiere a la idoneidad de los empleados de la DIAN, al mérito ni al papel que juega la Comisión Nacional del Servicio Civil (CNSC) como el ente encargado de la selección para el acceso a la carrera administrativa. Igualmente en el proyecto, se ratifica la dirección militar en los puestos de frontera, sin que previamente se haya hecho una evaluación de los resultados de esa política y por el contrario desconociendo el estudio que muestra que el costo beneficio de la Policía Fiscal y Aduanera (POLFA) ha sido negativo para la Dirección de Impuestos y Aduanas Nacionales (DIAN).

De todas formas no hay voluntad política del Gobierno nacional para un verdadero fortalecimiento de la DIAN en personal y sistemas, lo cual ayudaría a disminuir la alta evasión en IVA y renta, que cuesta al año 
alrededor de $\$ 26$ billones. Esta falta de voluntad política también se traduce en la ausencia de mecanismos efectivos para perseguir los capitales que se fugan hacia paraísos fiscales sin pagar impuestos, las mercancías subfacturadas que ingresan desde territorios como Panamá, arruinando a productores nacionales, y las estrategias que utilizan las transnacionales minero-energéticas para comercializar a menos precio los recursos naturales del país. La progresiva disminución de los aranceles como producto de los tratados de libre comercio también está erosionando los ingresos del Estado.

Finalmente, hay una cantidad de aspectos que no se consideran en la reforma, por lo cual no es posible hablar del cumplimiento de los principios constitucionales de equidad, proporcionalidad y progresividad, y mucho menos de los objetivos del discurso político de la actual reforma de la calidad tributaria, la lucha contra la evasión y llevar el recaudo al nivel potencial:

- Impuesto a las remesas empresariales: el Gobierno sigue sin restaurarlo, el cual, según los cálculos de Justicia Tributaria recaudaría cerca de $\$ 2,1$ billones y posibilitaría la reinversión de utilidades por parte de las empresas, generando así mayores puestos de trabajo o compra de insumos para sus actividades.

- Reforma con enfoque de género: aún no hay una explicación valedera para gravar con la tarifa general del IVA a productos básicos de uso femenino como las toallas higiénicas y los tampones, lo cual tiene un impacto severo sobre las finanzas de las mujeres en el país, teniendo en cuenta que ganan $20 \%$ menos que los hombres y tienen menos oportunidades de accesos al mercado laboral en Colombia.

- Financiación del SENA y el ICBF: la reforma tributaria de 2012 lesionó la financiación de estas dos importantes instituciones para la estabilidad y el desarrollo social del país, al disminuir los parafiscales y establecer el CREE, un impuesto con destinación específica. Los excedentes generados por este último no han sido trasladados a las instituciones indicadas, sino que han servido para generar unidad de 
caja en las necesidades del Gobierno nacional. Se estima que para el 2016 el SENA dejaría de recibir \$1 billón, que le corresponden del CREE, pero que están siendo apropiados irregularmente por el Gobierno.

- Lucha contra la evasión tributaria: a pesar de que las estimaciones de evasión en Colombia suman más $\$ 26$ billones de pesos (evasión en renta + IVA), los recaudos por gestión de la DIAN apenas llegan a máximo de $\$ 7$ billones en el 2022, una cifra ínfima comparado con lo que se pierde año a año, y para sumar la propuesta se encamina a sanciones laxas para valores superiores a los $\$ 5.000$ millones, facilitando que la anunciada cárcel para los evasores no se aplique.

\section{Conclusiones}

Las razones aquí esgrimidas muestran que esta reforma tributaria en nada contribuye a la solución de fondo de los problemas económicos y sociales del país, todo lo contrario. Se hace imperioso que el Congreso de la República tome cartas en el asunto, si -como lo expresa con frecuencia- quiere ver a un país menos desigual. La tributación no puede basarse en impuestos indirectos y regresivos que afectan la capacidad de gasto e inversión de los ciudadanos, ni que se disfrazan de directos, pero terminen afectando en mayor medida a las pequeñas y medianas empresas, principal fuente de generación de empleo del país.

La crisis económica del país tiene bases estructurales que no se resuelven con la reforma tributaria presentada al Congreso. Una verdadera justicia tributaria debería promover el fortalecimiento de fuentes de generación de riqueza en la agricultura, la industria y en los servicios de alto valor agregado, un gasto público efectivo y suficiente para las necesidades de desarrollo del país y, por supuesto, que los más ricos y las transnacionales paguen lo justo.

Las consecuencias de esta reforma tributaria que aumenta los impuestos indirectos y reduce los directos, en un contexto macroeconómico adverso 
de desaceleración económica, déficit fiscal y comercial, caída del consumo y aumento de la moratoria en los créditos, provocará una disminución del nivel de vida de los hogares de ingresos bajos y medios que conducirá a una profundización de la crisis económica del país. Por eso la mejor recomendación es no aprobarla.

\section{Referencias}

Cámara de Representantes. (23 de octubre de 2016).. Por medio del cual se dictan normas en materia tributaria y se dictan otras disposiciones. [Proyecto de Ley]. Colombia.

Congreso de la República. (30 de diciembre de 2016). Por medio de la cual se expiden normas en materia tributaria y se expiden otras disposiciones. [Ley 1819]. Colombia.

Corte Constitucional. (15 de julio de 2015). Sentencia C-726. [M.P. Gloria Stella Ortiz Delgado].

Dirección de Impuestos y Aduanas Nacionales. (2016). Estatuto tributario. Colombia.

Martínez, J., y Suárez, A. (2016). Desigualdad empresarial y Tarifa Única Tributaria. Bogotá: ACOPI.

Ministerio de Hacienda. (2015). Exposición de motivos del proyecto de ley: por medio de la cual se adopta una reforma tributaria estructural, se fortalecen los mecanismos para la lucha contra la evasión y la elusión fiscal, y se dictan otras disposiciones. Bogotá. Recuperado de http://www.minhacienda.gov. co/HomeMinhacienda/ShowProperty?nodeId=\%2FOCS\%2FP_MHCP_ WCC-059072\%2F\%2FidcPrimaryFile\&revision=latestreleased 